\title{
Optimal Supervisory Control of Two Phases Intersection Using Hybrid-based Model Approach
}

\author{
Bilal Tolbi \\ Department of Automatic, University of Sidi Bel-Abbes, BP 89, 22000, Sidi Bel-Abbes, Algeria. \\ LAIG, University of Guelma, BP401, 24000, Guelma, Algeria. \\ Received 29 February 2020; Accepted 2 October 2020
}

\begin{abstract}
In the present paper, a hybrid approach that combines modular modelling and Timed Petri Nets model is proposed, the main interest of this combination is to ensure optimal supervisory control of urban traffic flows in two phases intersections of medium-sized cities. Each intersection is considered as a multi-agent system, composed of all agents who represent all physical spaces. The behaviours of interconnected agents are supervised by the Timed Petri Nets model that controls the traffic light cycles according to an optimal timing plan defined by the Continuous Genetic Algorithm. A real two phases intersection in Sidi Bel-Abbes City is presented as an example. The proposed timing plan is compared with the current one using SUMO software for the most important objective functions. Also, the proposed model's behaviour is compared with TSIS-CORSIM software's model, and all obtained experiments results show the effectiveness of the approach and its possibility to be extended and applied to a many-phases intersection.
\end{abstract}

Keywords: CGA, MAS-based model, timed Petri net, supervisory control, timing plan.

\section{Introduction}

The rapid growth in transportation demand within the limited capacity in infrastructures, Urban Traffic Network (UTN) has become one of significant socio-economic issues in urban city life. Especially during last decades, the traffic congestion phenomenon has become predominant and drew great attention due to the world energy crisis and environmental worries. Different classical strategies try to reduce this phenomenon by the construction of new infrastructures, widening of busy roads, encouraging the use of public transport and implementing transport strategies (e.g. optimize the use of existing infrastructure). However, these solutions are still far for facilitate an optimal managing of traffic because they are unable to reply to the flow fluctuation in demand. In this purpose, the best solution for an optimal managing of the traffic congestion will be sought in the signal control strategy.

Recently, some researches propose a new view, the UTN is considered as Hybrid Dynamical System (HDS) [1]. In the case where the UTN is large, cycle length of system-wide becomes unsuitable by taking the network as a single system. Indeed, there are some researchers interested to divide UTN into single intersections and study each intersection separately see.eg [2]. When the number of intersections increases, more variables need to be determined and more constraints are imposed. Therefore the analytical control approaches as in [3] become not explicitly achievable. For this, other works propose optimisation program methods based on heuristic algorithms as in [4]. Other approaches integrate traffic simulation models in order to get the optimal coordinated timings for intersections as in [5].

\footnotetext{
*E-mail address: bilal.tolbi@univ-sba.dz

ISSN: $1791-2377$ @ 2020 School of Science, IHU. All rights reserved.

doi:10.25103/jestr. 135.14
}

The Multi-Agent System (MAS) has been provided on many applications from robotics engineering to economics and social sciences [6]. MAS consist of a set of dynamical autonomous agents interacting between each other to solve a complex task through collaboration. A representation of UTN systems using MAS is introduced in [7]. We can also find in the literature, approaches that apply Petri Nets (PNs) in UTN modelling, simulation or optimization [8]. The research works presented in [9] use timed and colored PNs to describe UTN, where the token represent the vehicle position. The initial attempts which have used Hybrid Petri Nets (HPNs) model can be found in [10]. The combination of Petri-nets and real-time multi-agent systems, for agent's communication modelling, is introduced in [11].

It's proved in [12] that the traffic light control system is one of the most profitable solutions for transportation problems, by improving the policies that control traffic lights. Urban traffic flows can be improved for a considerably lower cost, by minimizing the total delay at intersection [13]. We introduce several selected methods optimization-based signal traffic control for single intersection: Webster method [14], some heuristic or metaheuristic methods [15], differential evolution bacteria foraging algorithm [16], and Genetic algorithm [17]. For this context, this paper is devoted.

Moreover, some traffic researchers have done an effort to develop several simulation platforms to evaluate different traffic models and/or control UTN flows, most of them use graphic editors to draw, simulate and analyze the UTN capacity, maximize green wave band or minimize the delay, we can site as example: TSIS-CORSIM (Traffic Software Integrated System, CORridor SIMulation) [11] AIMSUN and SUMO (Simulation of Urban MObility), MAXBAND, TRANSYT-7F, SYNCHRO, SCOOT, OPAC...etc.

The main objective of this paper is to give an efficient timing plan of TPNs that control a two phases intersection taken from UTN of medium-sized city; the TPNs are 
combined with agents of MAS in hybrid architecture. Each physical space of the intersection represents an agent, and the behaviour of the interconnected agents is supervised by the TPNs. Thus, the following points address the main contributions of this paper:

- Physical spaces are described with modular differential equations where a flow of vehicles can be defined. This model includes the effects of the traffic lights, the street and intersection saturation levels, the change of vehicle flows due to the quantity of cars and the congestion phenomena by the interconnections of the agents.

- The traffic light cycles are defined by TPN model, with the supervising possibility of these cycles.

- To estimate the delay of vehicles, a new timing plan, different to the current one, is optimized and proposed, using Continuous Genetic Algorithm, to represent transitions durations of the TPN model.

- The proposed plan is compared with the current one, fixed by the city's municipal authority, on SUMO software under different objective functions.

- The proposed hybrid model is compared with TSISCORSIM software's model on Matlab, in order to show the scalability of the approach.

The remainder of the paper is organized as follows: section II gives the general definition of TPNs. The problem statement is presented in section III, the hybrid model presentation for urban traffic flows control in a single intersection is presented in section IV. An example of real single intersection in medium-sized city is supplied in section V. Section VI presents the timing plan problem and its solution. The obtained experiments results are given in section VII. Finally, future work and conclusions are given in section VIII.

\section{Timed PETRI NETS Definition}

A Timed Petri-Net TPN; is a graph structure defined by the following six-tuple

$T P N=\left(P, T, A, w, M_{0}, \delta\right)$

$P=\left\{p_{1}, p_{2}, \ldots p_{n}\right\}$ is a finite set of $n$ nodes in a graph, called Places.
$T=\left\{t_{1}, t_{2}, \ldots t_{m}\right\}$ is a finite set of $m$ nodes called Transitions.

$A: A \subseteq(P \times T) \cup(T \times P)$ is a finite set of arcs; they connect transitions and places.

$w: A \rightarrow \mathbb{Z}^{+}$is the positive integer weights of arcs.

$M_{0}$ is the initial marking or number of tokens contained in each place $p \in P$.

Let $M_{t m}(p): P \rightarrow \mathbb{Z}^{+}$defines the $n$-th vector with tokens number in each place at time $t m$. The $t m$-th PN marking is achieved according to the transition rule bellow. A transition $t_{i} \in T$ is said to be enabled in a PN with finite capacity if and only if:

$$
\begin{aligned}
& M_{t m}\left(p_{j}\right) \geq w\left(p_{j}, t_{i}\right), \forall p_{j} \mid w\left(p_{j}, t_{i}\right) \in w, \text { such as } j=1, \ldots ., n ; \\
& i=1, \ldots, m .
\end{aligned}
$$

If $t_{i}$ is enabled the next marking is defined as:

$$
M_{t m+1}\left(p_{j}\right)=M_{t m}\left(p_{j}\right)-w\left(p_{j}, t_{i}\right)+w\left(t_{i}, p_{j}\right) .
$$

$\delta: T \rightarrow \mathbb{R}^{+}$is the finite set of transitions time delays, each transition $t_{i}$ in the net assigned by positive number $\tau_{i}$ such as: $\tau_{i}=\delta\left(t_{i}\right)$.

\section{Problem Statement}

Let $\beta=\beta_{s t} \cup \beta_{c r} \cup \beta_{s o} \cup \beta_{s i}$ is the set of agents or physical spaces where the vehicles can be moved or placed in a UTN. $\beta_{s t}=\left\{\beta_{s t}^{1}, \ldots ., \beta_{s t}^{n}\right\}$ is the set of $n$ street agents, $\beta_{c r}=\left\{\beta_{c r}^{1}, \ldots, \beta_{c r}^{m}\right\}$ is the set of $m$ intersection agents, $\beta_{s o}=\left\{\beta_{s o}^{1}, \ldots, \beta_{s o}^{k}\right\}$ is the set of vehicle sources, i.e. buildings, parkings, garages...etc. $\beta_{s i}=\left\{\beta_{s i}^{1}, \ldots ., \beta_{s i}^{s}\right\}$ is the set of vehicle sinks i.e. agents containing vehicles that leave the UTN. These allow us to construct all differential equations, explained below. A real example of a single intersection that illustrates all of these physical spaces is given in Figure 1, where the inputs and outputs of vehicles flow are respectively depicted by source and sink agents.

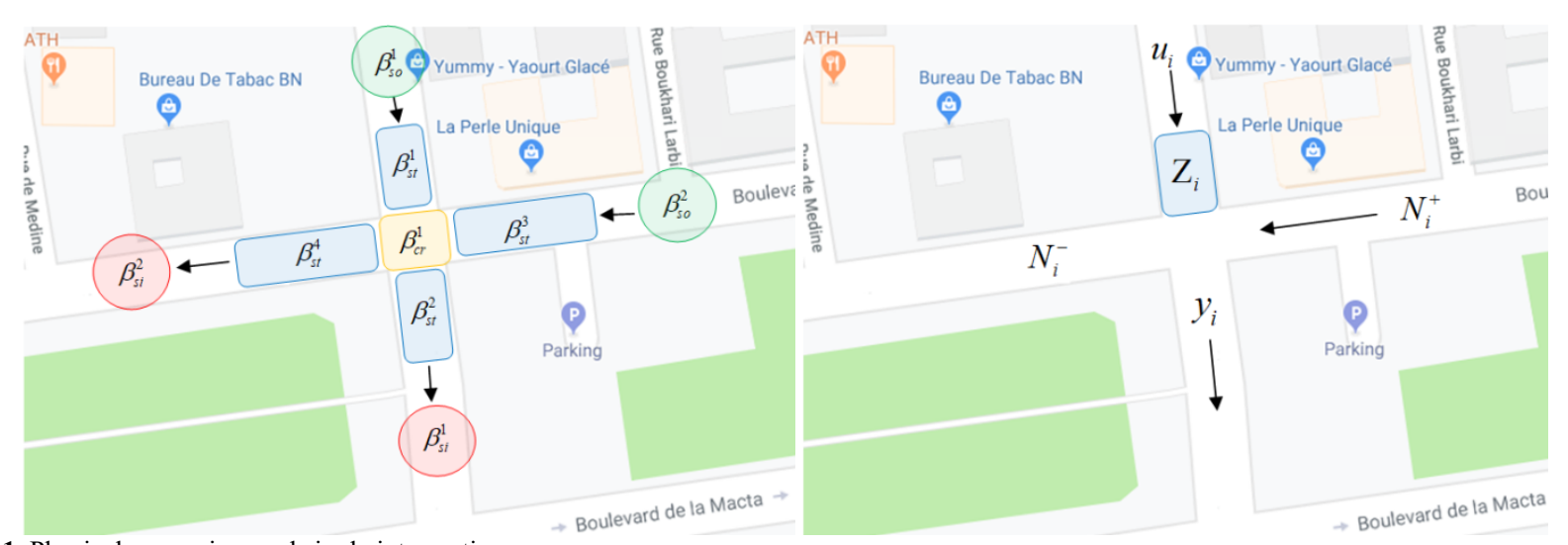

Fig. 1. Physical spaces in a real single intersection 
Let $N_{i}^{+} \subset \beta$ and $N_{i}^{-} \subset \beta$ are the in-adjacency and the outadjacency sets of $\beta_{i}$, which represent all physical spaces providing and carrying out vehicles to and from $\beta_{i} \in \beta$ respectively. Streets, intersections, sources and sinks interconnections are assured using these two sets. $z_{i} \in \mathbb{R}$ is the number of vehicles in each agent $\beta_{i} \in \beta, \alpha\left(z_{i}\right) \in \mathbb{R}, \forall \beta_{i} \in \beta$ is the vehicles speed function that represents the inflow and outflow rates according to vehicles density change in each agent. Thus the hybrid model that will allows us to control vehicle density and distribution, and the optimal traffic lights, is consisting of:

- A modular and simple flow differential equations set for each $z_{i}$ describing agent's occupation.

- A functions set $\alpha\left(z_{i}\right)$ to describe experimental data flows.

- A TPN set $\mathrm{PN}_{i}, \forall \beta_{c r}^{i} \in \beta_{c r}$ for traffic light management.

- A supervisory control is guaranteed using the relationships set, between the TPN models and the differential equations is to feedback loop characterization.

\section{Hybrid Model Presentation}

This section gives a modular and mathematical representation of UTN, by developing a differential equations set that represent all the agents in the intersection. This will permit to get easier the complex UTN encompassing all intersection variants. According to section III, the modular model of vehicle flow for each agent, can be given by:

$$
\begin{aligned}
& \dot{z}_{i}=\sum_{\beta_{i} \in N_{i}^{+}} u_{j, i}-\sum_{\beta_{k} \in N_{i}^{-}} y_{i, k} \\
& y_{i, k}=a_{i, k}\left(z_{k}\right) \Gamma_{i, k} \alpha_{i}\left(z_{i}\right) z_{i}, \quad \forall \beta_{k} \in N_{i}^{-}
\end{aligned}
$$

We put $z_{i}$ as the vehicles quantity in $\beta_{i} \in \beta, u_{j, i}$ and $y_{i, k}$ are the input flow to agent $\beta_{i}$ from $\beta_{j} \in N_{i}^{+}$and the output flow from $\beta_{i}$ to agent $\beta_{k} \in N_{i}^{-}$respectively. Therefore, and according to the equation (2), the $z_{i}$ dynamics are controlled by $u_{j, i}$ and $y_{i, k}$.

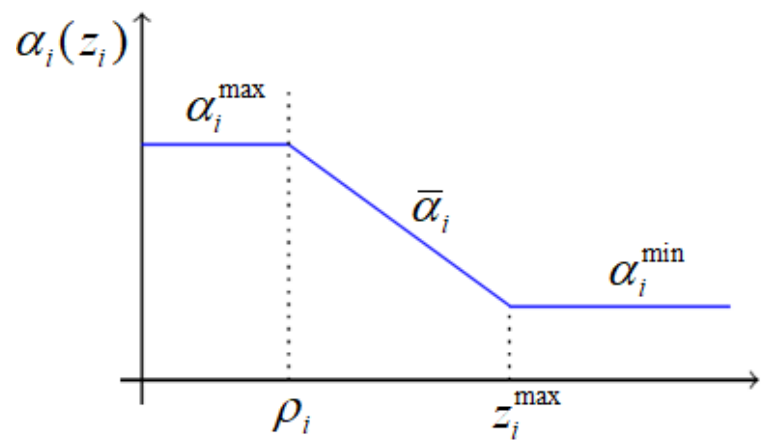

Fig. 2. The $\alpha_{i}$ change's with respect to $z_{i}$
From equation (3), $\alpha_{i}\left(z_{i}\right)$ is a function that expresses the vehicles unloading flow (Figure 2), it can be detailed as:

$\alpha_{i}\left(z_{i}\right)= \begin{cases}\alpha_{i}^{\max }, & \text { if } z_{i}<\rho_{i} \\ \bar{\alpha}_{i}, & \text { if } \rho_{i}<z_{i}<z_{i}^{\max } \\ \alpha_{i}^{\min }, & \text { if } z_{i}=z_{i}^{\max }\end{cases}$

From Figure $2, z_{i}^{\max }$ is the vehicles maximum density for each agent, we can define the linear decrease of $\alpha_{i}$ as:

$\bar{\alpha}_{i}=\left(\frac{a_{\min }-\alpha_{i}^{\max }}{z_{i}^{\max }-\rho_{i}}\right)\left(z_{i}-\rho_{i}\right)+\alpha_{i}^{\max }$

Each agent is given by the physical length of streets, according to a minimum value $\alpha_{i}^{\min }<\alpha_{i}^{\max }$. The value of $\alpha_{i}^{\max }$ depends on the area of the agent $\beta_{i}$. If the number of vehicles in the agent increases, then the rate of unloading flow will decrease in this case. We can find many similar functions in the literature, as in [18]. Also, we define the activation functions $\Gamma_{i, k}$ and $a_{i, j}$ which activate and deactivate the output flows, $\Gamma_{i, k}$ is given by:

$\Gamma_{i, k}=\left\{\begin{array}{cl}c_{i, k} M_{k}, & \text { if } \beta_{k} \in \beta_{c r} \\ c_{i, k} M_{i}, & \text { if } \beta_{i} \in \beta_{c r} \\ 1, & \text { otherwise }\end{array}\right.$

such as $M_{k} \in \mathbb{R}^{n_{k} \times 1}$ is the actual marking vector of the TPN with $n_{k}$ places representing the traffic light behaviours for the single intersection $\beta_{k}$, and $c_{i, k} \in \mathbb{R}^{1 \times n_{k}}$ correspond to the traffic light cycle of each phase. Thus, the function $\Gamma_{i, k}$ is related to a traffic light cycle if the output $y_{i, k}$ connects the agent $\beta_{i}$ to a single intersection $\beta_{k}$ supervised by a traffic light, and it is obtained as a linear combination of the vector $M_{k}$. If there is no traffic light for $\beta_{k}$ or $\beta_{k} \notin \beta_{c r}$, then $\Gamma_{i, k}=1$ i.e. always activated, thus a free flow of vehicles.

The feedback information is represented by the functions $a_{i, k}\left(z_{k}\right)$ in order to control the output flow of $\beta_{i}$ depending on the saturation level of the downstream agent $\beta_{k} \in N_{i}^{-}, a_{i, k}\left(z_{k}\right)$ is defined as:

$a_{i, k}\left(z_{k}\right)=\frac{1}{1+\exp \left(k_{e}\left(\frac{z_{k}}{z_{k}^{\max }}-\xi\right)\right)}$

such as $k_{e}, \xi \in \mathbb{R}$. When $\beta_{k}$ is full, $a_{i, k} \approx 0$ and the output flow of the precedent agent $\beta_{i}$ is blocked. Finally, the equations (2) 
and (3) are applied directly to streets and intersections i.e. $\forall \beta_{i} \in \beta_{s t}, \beta_{c r}$, also to sources and sinks i.e. $\beta_{i} \in \beta_{s o}, \beta_{s i}$

The flows switching is assured by adding TPN to MASbased model in the context of control theory, according to the shame shown in Figure 3. Depending on certain traffic light cycle, the functions $\Gamma_{i, j}$ are activated by TPN and the feedback signals $a_{i, j}$ are transmitted from the MAS-based model. This communication ensures the supervisory control of the single intersection. The traffic light cycle has been defined by applying an optimization method to get the optimal timing plan. The timing optimization problem is presented in section VI. A real example of two phases intersection is presented immediately to illustrate the approach.

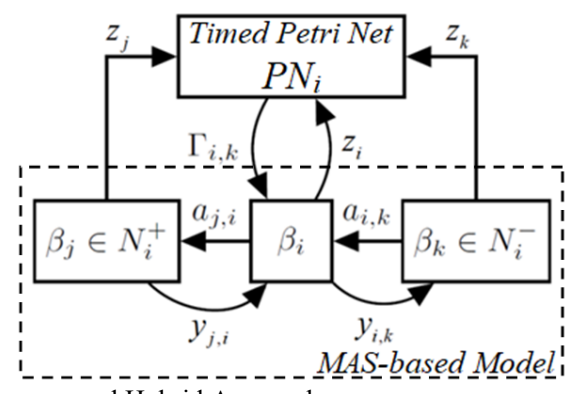

Fig. 3. The proposed Hybrid Approach

\section{Case Study}

The modular model of vehicle flow obtained in the previous section, is applied to a real intersection in Sidi Bel-Abbes, as an example of medium-sized city in Algeria, the intersection of Colonel Amirouch Ave and Mohammed Khmista Ave is composed by a simple four-road, with one bidirectional and one unidirectional, as shown in Figure 4. The studied intersection is fed by three vehicle sources and discharged by three sinks; it admits two phases signal control as illustrated in Figure 5. Using the formulation described in section IV, the intersection is redrawn as shown in Figure 6. Thus, $n=6$, $m=1, k=3, \quad s=3$ so that, the subscripts are reordered: $\beta_{s t}=\left\{\beta_{1}, \beta_{2}, \beta_{3}, \beta_{4}, \beta_{5}, \beta_{6}\right\}, \quad \beta_{c r}=\left\{\beta_{7}\right\}, \quad \beta_{s o}=\left\{\beta_{9}, \beta_{11}, \beta_{12}\right\}$ and $\beta_{s i}=\left\{\beta_{8}, \beta_{10}, \beta_{13}\right\}$. Since $\beta_{7}$ has traffic light, a corresponding TPN $\mathrm{PN}_{7}$ is set. According to the agents' interconnection shown by the arrows in Figure 6, the input and output adjacency subsets for agent $\beta_{7}$ (as example) can be given by:

$$
N_{7}^{+}=\left\{\beta_{1}, \beta_{3}, \beta_{5}\right\}, N_{7}^{-}=\left\{\beta_{2}, \beta_{4}, \beta_{6}\right\}
$$
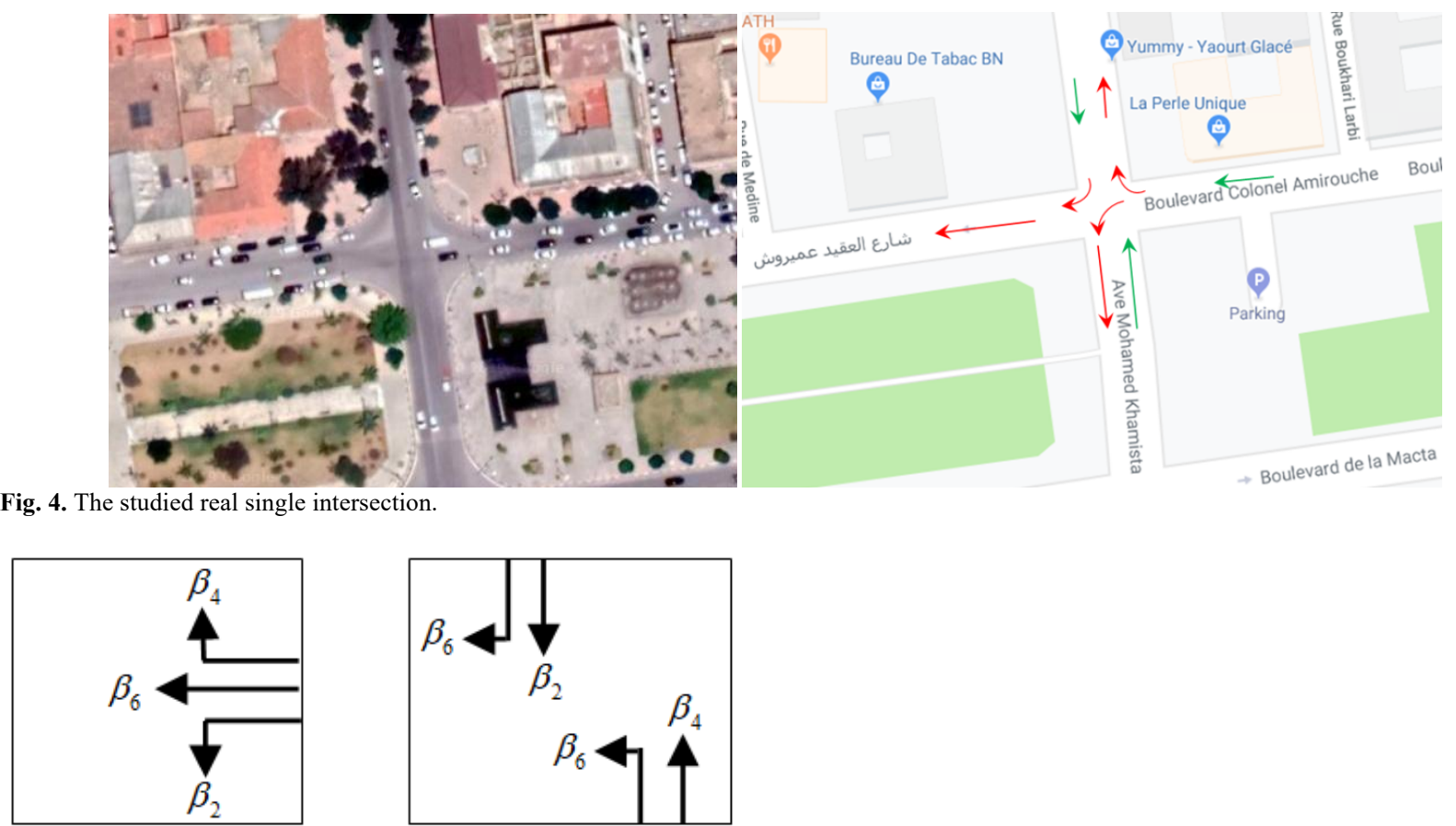

Fig. 4. The studied real single intersection.

Phase 1

Fig. 5. The two phases of studied intersection.

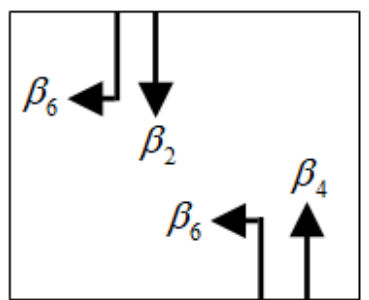

Phase 2 


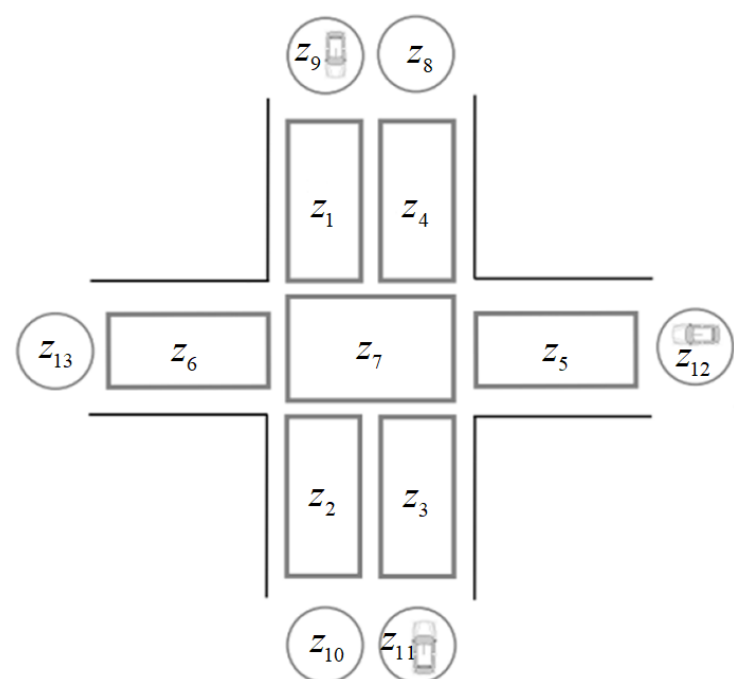

Fig. 6. The agents interconnection in the studied intersection

Therefore, outputs $y_{i, j}$ becomes inputs $u_{i, j}$ when we are interested by $\beta_{j}$ (eg. $y_{1,7}=u_{1,7}$ ), thus the equations set of each agent (eg. $\beta_{6}$ and $\beta_{7}$ ) is defined bellow, by applying the equations (2) and (3):

$\beta_{6}:\left\{\begin{array}{l}\dot{z}_{6}=u_{7,6}-a_{6,13}\left(z_{13}\right) \Gamma_{6,13} \alpha_{6}\left(z_{6}\right) z_{6} \\ y_{6,13}=a_{6,13}\left(z_{13}\right) \Gamma_{6,13} \alpha_{6}\left(z_{6}\right) z_{6}\end{array}\right.$

$\beta_{7}:\left\{\begin{aligned} \dot{z}_{7}= & u_{5,7}+u_{3,7}+u_{1,7}-\alpha_{7}\left(z_{7}\right) z_{7}\left[a_{7,4}\left(z_{4}\right) \Gamma_{7,4}\right. \\ & \left.+a_{7,2}\left(z_{2}\right) \Gamma_{7,2}+a_{7,6}\left(z_{6}\right) \Gamma_{7,6}\right] \\ y_{7,4}= & a_{7,4}\left(z_{4}\right) \Gamma_{7,4} \alpha_{7}\left(z_{7}\right) z_{7} \\ y_{7,2}= & a_{7,2}\left(z_{2}\right) \Gamma_{7,2} \alpha_{7}\left(z_{7}\right) z_{7} \\ y_{7,6}= & a_{7,6}\left(z_{6}\right) \Gamma_{7,6} \alpha_{7}\left(z_{7}\right) z_{7}\end{aligned}\right.$

As long as there are no traffic lights, $\Gamma_{9,1}, \Gamma_{7,2}, \Gamma_{2,10}$ , $\Gamma_{11,3}, \Gamma_{7,4}, \Gamma_{4,8}, \Gamma_{12,5}, \Gamma_{7,6}$ and $\Gamma_{6,13}$ are always equal to one. The same thing for the saturation functions $a_{4,8}, a_{2,10}$ and $a_{6,13}$, they are equal to one at all time, since the maximal values $z_{8}^{\max }, z_{10}^{\max }, z_{13}^{\max }$ are infinite. The TPN $\left(\mathrm{PN}_{7}\right)$ that represents the traffic light cycle of each phase is shown in Figure.7. The green, yellow and red lights are, respectively, represented by the places $\left\{p_{1}, p_{2}, p_{3}\right\}$ which control the vehicles flow coming from agent $\beta_{1}$ to $\beta_{7}$. Also, $\left\{p_{4}, p_{5}, p_{6}\right\}$ control the vehicles flow coming from street agent $\beta_{3}$ to $\beta_{7}$, while $\left\{p_{7}, p_{8}, p_{9}\right\}$ control the vehicles flow coming from the street agent $\beta_{5}$ to $\beta_{7}$. Note that $M_{0}=[1,0,0,1,0,0,0,0,1]$ is initial marking of the TPN i.e. $\left\{p_{1}, p_{4}, p_{9}\right\}$ have one token, that means:

1 - The green light is "on", there is a flow coming from $\beta_{1}$ and $\beta_{3}$ to $\beta_{7}$ (phase 2), so $\Gamma_{1,7}=\Gamma_{3,7}=1$

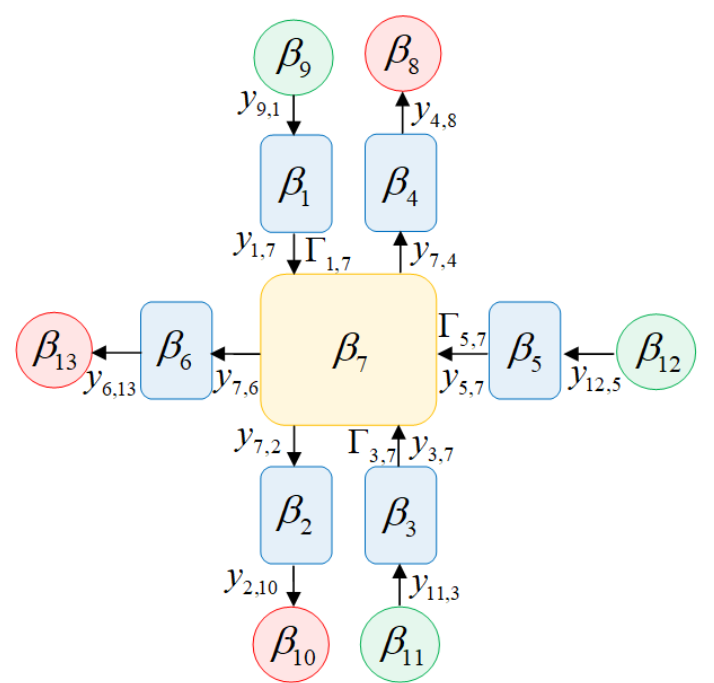

2- The red light is "on", there is no flow coming from $\beta_{5}$ to $\beta_{7}$ (phase 1 ), so $\Gamma_{5,7}=0$.

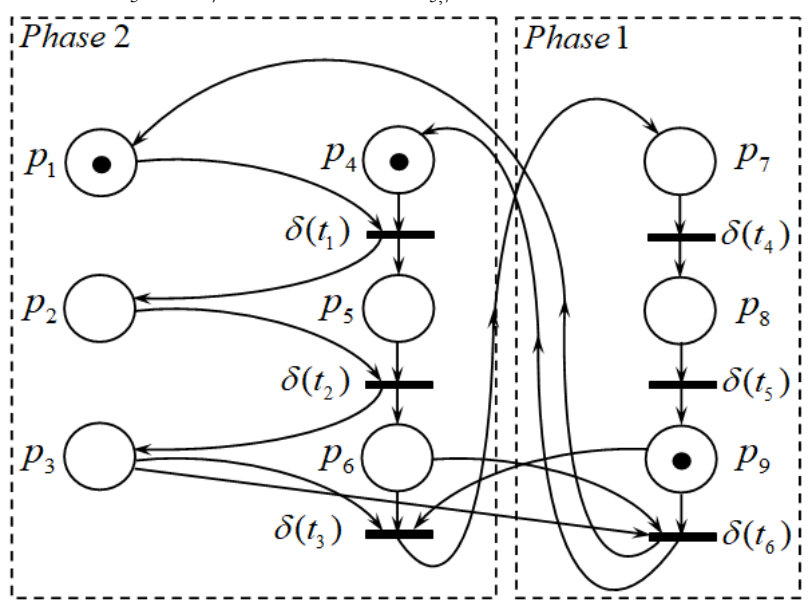

Fig. 7. Timed PN for the studied intersection

It's important that the optimal choice of traffic light cycle, which is represented by the set of $\delta\left(t_{i}\right)$, leads to optimal activation of functions $\Gamma_{i, j}$, thus it gives an optimal supervisory control of intersection and allows avoiding congestion. The second problem presented here is an optimization problem, that's why the following section is devoted.

\section{Timing Optimization Problem}

Firstly, we give bellow, the most used terminology and notions for a signal traffic intersection:

- $C=$ cycle length $(\mathrm{sec})$

- $c=$ capacity rate (veh/sec, or veh/cycle, or veh/h)

- $d$ = average delay ( $\mathrm{sec}$ )

- $d_{u n}=$ average uniform delay (sec)

- $d_{o v}=$ average overflow delay $(\mathrm{sec})$

- $g_{i}=$ effective green time (sec)

- $G=$ displayed green time (sec)

- $h=$ time headway (sec)

- $q=$ arrival flow rate $(\mathrm{veh} / \mathrm{sec})$

- $Q 0=$ expected overflow queue length (veh)

- $Q(t)=$ queue length at time $\mathrm{t}$ (veh) 
- $r$ = effective red time $(\mathrm{sec})$

- $R=$ displayed red time $(\mathrm{sec})$

- $S=$ departure (saturation) flow rate from queue during effective green $(\mathrm{veh} / \mathrm{sec})$

- $t=$ time

- $T d=$ duration of analysis period in time dependent delay models

- $x$ = degree of saturation, $x=(q / S) /(g / c)$, or $x=q / C$

- $y=$ flow ratio, $y=q / S$

- $Y=$ yellow (or clearance) time (sec)

In this work, we assume that " $\mathrm{C}$ " is not constant over time and is not priori unknown unlike in the work [17] where the authors consider the cycle length is constant over time and prior known, whether traffic demand. Where this hypothesis is restricted, however, lengths of particular phases (i.e., green times) can change over time; they are the decision variables in our optimal control. For this, delay is minimized according to the following equations of the U. S. Highway Capacity Manual (2000):

$$
\sum_{j=1}^{J} d_{j, T d}
$$

$d=d_{u n} * D F+d_{o v}$

With:

$d_{u n}=\frac{C(1-g / C)^{2}}{2(1-\mathrm{xg} / \mathrm{C})}$

$$
d_{o v}=900 f l x^{2}\left[(x-1)+\sqrt{(x-1)^{2}+\frac{m x}{c f l}}\right]
$$

Where $D F$ is the delay factor to account for signal coordination and controller type; $m$ is the calibration parameter which depends on the arrival pattern and $f l$ is the flow period in hours $(f l=0.25$ in $1994 \mathrm{HCM})$. Finally, the timing optimization problem for model (11) is formulated as following:

$$
\underset{C, g_{i}, T d}{\operatorname{Minimize}}\left(\sum_{i=1, \ldots, I}^{J} d_{j, T d}\right)
$$

Such as:

$$
\begin{aligned}
& \sum_{i=1}^{I} g_{i, T d}=C \\
& g_{i, T d} \geq g_{i, \min }, \quad g_{i, T d} \leq g_{i, \max } \\
& C \geq C_{\min }, \quad C \leq C_{\max } \\
& g_{i, \max }<C
\end{aligned}
$$

Where: $g_{i, \min }$ is the minimum green time(s); $g_{i, \max }$ is the maximum green time(s); $C_{\min }$ is the minimum cycle length time(s); $C_{\max }$ is the maximum cycle length time(s); in equation (12) the function is non-linear and non-convex, that makes it difficult to obtain the best and the global minimums. In our case these minimums are obtained using Continuous Genetic Algorithm (CGA) [19], it is an adaptation of Genetic Algorithm (GA) to the continuous optimization problems.

Especially, CGA uses a type of real coding, which is as close as possible to Hollands approach using binary coding. The algorithm starts with an initial population of " $n$ ", individuals: an individual is composed of real coordinates, respectively associated to the variables of the objective function at hand. The reproduction operators, inspired by the genetics, are applied to this population; offspring are created from parents. The new population is constituted in selecting the best individuals. By repeating this process, one hopes to enrich gradually the population with the most efficient individuals. The usual mechanisms of reproduction are the crossover, which consists in exchanging some coordinates of two individuals, and the mutation, which consists in generating a new coordinate at a given place of one individual.

CGA allows us to resolve the timing optimization problem, each individual in CGA stands for a timing scheme of the intersection, as, $g_{i}=\left\{g_{1} ; g_{2} ; \ldots ; g_{I}\right\}$ in which $g_{i}$ represents real green time of phase $i$ in the scheme and it is the variable remaining to be optimized according to general steps of the algorithm presented bellow.

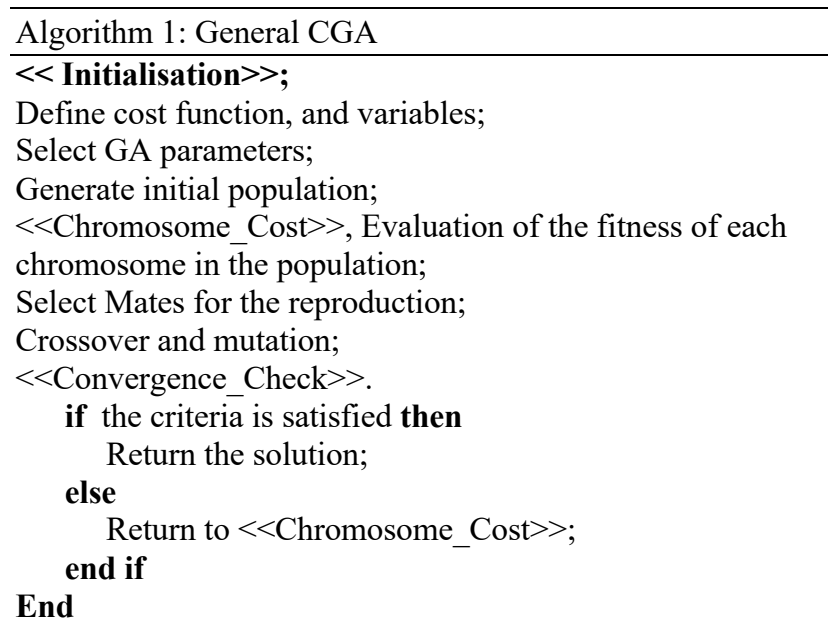

\section{Experiments Results}

The hybrid model is built on Matlab, and the data are simulated using SUMO and TSIS-CORSIM Software. The real data were collected from the real single intersection (section $\mathrm{V}$ ), where the sources $\beta_{9}, \beta_{11}, \beta_{12} \in \beta_{\text {so }}$ provide approximately 700, 953 and $140(\mathrm{veh} / \mathrm{h})$ respectively. The street agents $\beta_{1}, \beta_{2}, \beta_{3}, \beta_{4}, \beta_{5}, \beta_{6} \in \beta_{s t}$ and $\beta_{7} \in \beta_{c r}$ are initially empty. The maximum occupancy of vehicles in each agent $\beta_{i} \in \beta_{s t}$ is defined as: $z_{1}^{\max }=110, \quad z_{2}^{\max }=46, \quad z_{3}^{\max }=46$, $z_{4}^{\max }=110, z_{5}^{\max }=17, z_{6}^{\max }=20$ and $z_{7}^{\max }=22$, in the case of sources $z_{9}^{\max }=800, z_{11}^{\max }=1050, z_{12}^{\max }=240$. According to the Current Traffic Signal Control Plan (CTSCP) which is fixed by municipal authority of Sidi Bel Abbes city, time 
delays of $\mathrm{PN}_{7}$ (Figure 7) are given by $\delta\left(t_{1}\right)=35, \delta\left(t_{4}\right)=45$, $\delta\left(t_{2}\right)=\delta\left(t_{5}\right)=5$ and $\delta\left(t_{3}\right)=\delta\left(t_{6}\right)=5$, so that $g_{l}=45 \mathrm{sec}$ and $g_{2}=35 \mathrm{sec}$ (green light of phase1 and phase 2). CTSCP and our Proposed Traffic Signal Control Plan (PTSCP) are simulated using SUMO software. The obtained results are presented in the Table 1 . The Table 2 shows the parameters of the vehicles such as waiting time, loss time, $\mathrm{CO} 2$ and $\mathrm{CO}$ emission rate, NOx nitrogen oxide and noise under PTSCP and CTSCP. According to Table 2 the results shows that that PTSCP surpass clearly the CTSCP for all objective functions.

Table 1. PTSCP and CTSCP of green Lights

\begin{tabular}{c|c|c}
\hline \multirow{2}{*}{ Phases } & \multicolumn{2}{|c}{ Phases duration (sec) } \\
\cline { 2 - 3 } & PTSCP & CTSCP \\
\hline Phase 1 & 20 & 35 \\
Phase 2 & 40 & 45 \\
Cycle length & 60 & 80 \\
\hline
\end{tabular}

Table 2. Numerical Comparison between PTSCP and CTSCP

\begin{tabular}{c|c|c|c}
\hline $\begin{array}{c}\text { Objective } \\
\text { functions }\end{array}$ & PTSCP & CTSCP & $\begin{array}{c}\text { Improvement } \\
(\%)\end{array}$ \\
\hline Waiting time & 31 & 54 & 42.59 \\
(sec) & & & \\
Time loss (sec) & 38 & 85 & 55.29 \\
CO2 (mg/sec) & 3986.31 & 6081.51 & 34.45 \\
CO (mg/sec) & 65 & 112 & 41.96 \\
NOx (mg/sec) & 1.79 & 2.72 & 34.19 \\
Noise (db) & 46.13 & 49.41 & 6.63 \\
\hline
\end{tabular}

Figures 8 to 10 presents the simulation results of agents behaviours obtained from Matlab. Figure 8 describes the number of vehicles changing with respect to time in the street agents $\beta_{1}, \beta_{3}, \beta_{5} \in \beta_{s t}$ i.e. the input flow coming from $\beta_{9}, \beta_{11}, \beta_{12} \in \beta_{\text {so }}$ respectively. Figure 9 and 10 , present the behaviour of intersection agent, $\beta_{7} \in \beta_{c r}$ and the occupancy of $\beta_{2}, \beta_{4}, \beta_{6} \in \beta_{s t}$ respectively. According to the obtained results shown in these figures, we can observe that all agents $\beta_{i}$ do not saturate due to our sequence of PTSCP (Table 1) of traffic lights.
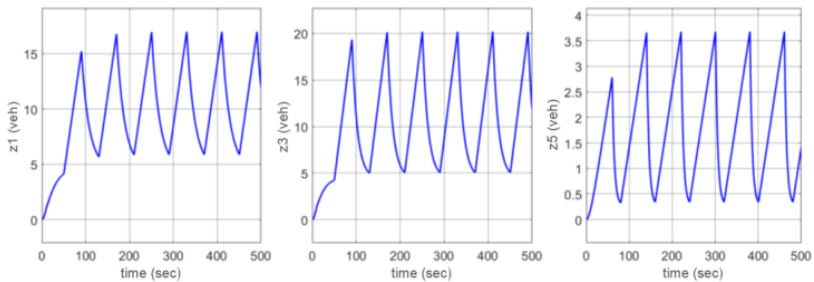

Fig. 8. The behaviour of agents $\beta_{1}, \beta_{3}, \beta_{5} \in \beta_{s t}$.

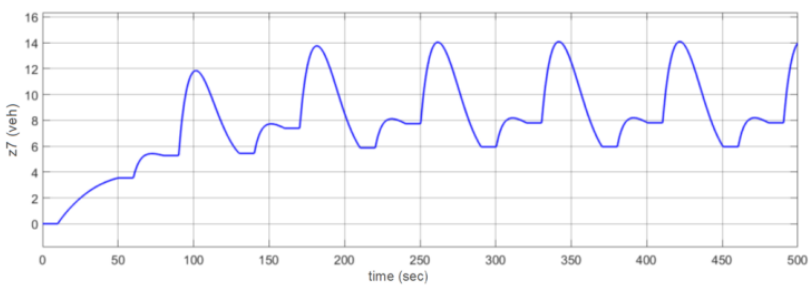

Fig. 9. The behaviour of agent $\beta_{7} \in \beta_{c r}$.
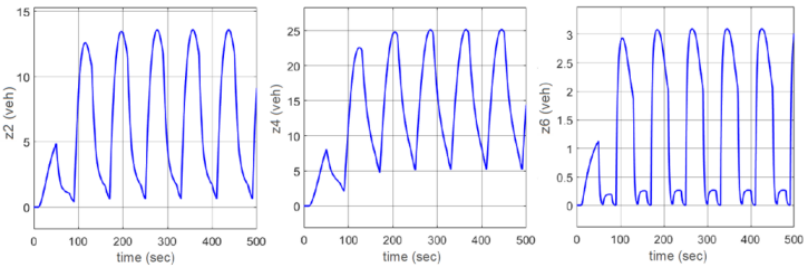

Fig. 10. The behaviour of agents $\beta_{2}, \beta_{4}, \beta_{6} \in \beta_{s t}$.

Another simulation of the studied example was carried out to formalize the hybrid approach through the simulation software package, TSIS-CORSIM. The figures below were obtained using the same real data. The input and output flows provided by street agents $\beta_{1}, \beta_{3}, \beta_{5} \in \beta_{s t}$ and $\beta_{2}, \beta_{4}, \beta_{6} \in \beta_{s t}$ of the hybrid-based model approach, are compared with TSISCORSIM software's flows and the results are illustrated by red graph and bleu one respectively on Figure 11 and Figure 12 .

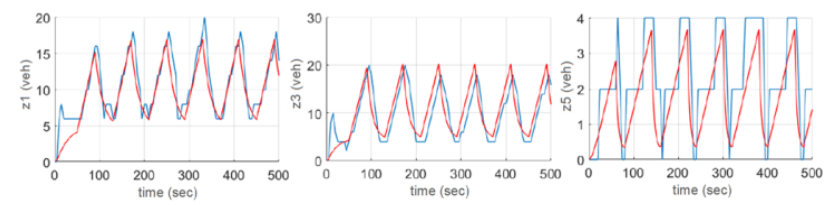

Fig. 11. The input flows from $\beta_{1}, \beta_{3}, \beta_{5} \in \beta_{s t}$.

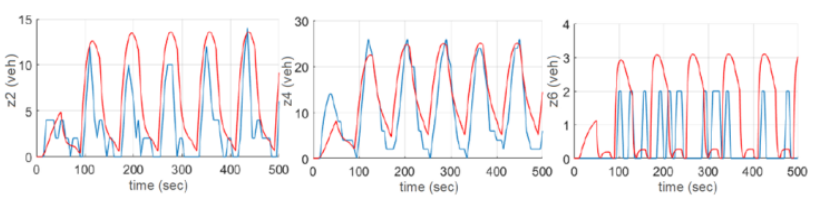

Fig. 12. The output flows to $\beta_{2}, \beta_{4}, \beta_{6} \in \beta_{s t}$.

\section{Conclusions}

We have presented in this paper an optimal supervisory control of a two phases intersection, taken from UTN of medium-sized city, through the TPN that control the single intersection. The TPN is combined with agents of MAS in hybrid architecture and it supervises effectively the behaviour of the interconnected agents due to the efficient timing plan (PTSCP) of the green lights. The green lights of each intersection phase optimized by CGA gives to the TPN an optimal performance to control traffic lights and, with consequence, intersection traffic flows, thus the network flows optimization.

This approach has been applied on a real single intersection chosen from the UTN of Sidi Bel-Abbes city because of its large capacity of sources, streets and sinks. The effectiveness and the scalability of the approach have been proven through the following two steps:

- $\quad$ The efficacy of the proposed timing plan (PTSCP) defined by CGA has been proven by comparing this plan with the current one (CTSCP) using SUMO software, under different objective functions.

- The results of the proposed hybrid model have been proved similar by comparing with the results of TSIS-CORSIM software's model.

Future work includes the development and simulation of a complex many-phases intersection in the same city network 
and further experiments on SUMO and TSIS-CORSIM with real data to optimize the flows and improve the most important objective functions.

\section{Acknowledgment}

We gratefully acknowledge the financial support from Algerian DIRECTORATE GENERAL FOR SCIENTIFIC RESEARCH AND TECHNOLOGICAL DEVELOPMENT (la DGRSDT).
This is an Open Access article distributed under the terms of the Creative Commons Attribution License

\section{References}

[1] B Tolbi, H Tebbikh, Hassane Alla. Fault-tolerant continuous flow systems modelling. International Journal of Systems Science, Taylor \& Francis, 2017, 48 (1), pp.107117. $\langle 10.1080 / 00207721.2016 .1160454\rangle$. 〈hal-01412952〉

[2] W. Xiang, J. Xiao, Y. Jiang, Real-time signalization for an oversaturated intersection via static state feedback control: A switched system approach, Journal of the Franklin Institute 352 (8) (2015) 3304\{3324. doi:10.1016/j.jfranklin.2014.09.017. URL http://www.sciencedirect.com/science/article/pii/S001600321400 2725

[3] K. Aboudolas, M. Papageorgiou, and E. Kosmatopoulos, "Store-andforward based methods for the signal control problem in largescale congested urban road networks," Transp. Res. Part C Emerg. Technol., vol. 17, no. 2, pp. 163-174, Apr. 2009.

[4] A. Hajbabaie and R. F. Benekohal, "A Program for Simultaneous Network Signal Timing Optimization and Traffic Assignment," vol. 16, no. 5, pp. 2573-2586, 2015.

[5] J. Byun and J. J. Kim, "Case Study of Queue Growth Equity Method for Urban Traffic Signal Optimization,” no. June, pp. 1-13, 2015.

[6] R. L. Axtell, " 120 million agents self-organize into 6 million firms: A model of the u.s. private sector," in Proceedings of the 2016 International Conference on Autonomous Agents \& Multiagent Systems, Richland, SC, 2016, pp. 806-816.

[7] B. Chen and H. H. Cheng, "A review of the applications of agent technology in traffic and transportation systems," IEEE Transactions on Intelligent Transportation Systems, vol. 11, no. 2, pp. 485-497, June 2010.

[8] K. M. Ng, M. B. I. Reaz, and M. A. M. Ali, "A review on the applications of Petri nets in modeling, analysis, and control of urban traffic," IEEE Transactions on Intelligent Transportation Systems, vol. 14, no. 2, pp. 858-870, June 2013.

[9] A. Di Febbraro, D. Giglio, and N. Sacco, "A deterministic and stochastic Petri net model for traffic-responsive signaling control in urban areas," IEEE Transactions on Intelligent Transportation Systems, pp. 1-15, 102015.

[10]L. Ghomri and H. Alla, "Modeling and analysis using hybrid Petri nets," Nonlinear Anal. Hybrid Syst., vol. 1, no. 2, pp. 141-153, Jun. 2007.
[11] Awais Qasim, Sidra Kanwal, Adnan Khalid, Syed Asad Raza Kazmi and Jawad Hassan, "Timed-Arc Petri-Nets based Agent Communication for Real-Time Multi-Agent Systems" International Journal of Advanced Computer Science and Applications(IJACSA), 2019. http://dx.doi.org/10.14569/IJACSA.2019.0100970

[12]Pedro Mercader, Melkior Ornik, Per-Olof Gutman, Ilya Ioslovich, Optimal Signal Timing for Multi-Phase Intersections,IFACPapersOnLine,Volume 51, Issue 9,2018,Pages 476-481.

[13] Hua Wei, Guanjie Zheng, Vikash Gayah, and Zhenhui Li. 2019. A Survey on Trafe Signal Control Methods. 1, 1 (July 2019), 31 pages. https://doi.org/10.1145/

[14]Peter Koonce et al. 2008. Traffic signal timing manual. Technical Report. United States. Federal Highway Administration.

[15] Hayder Amer,1,Naveed Salman, Matthew Hawes, Moumena Chaqfeh, Lyudmila Mihaylova, and Martin Mayfield. An Improved Simulated Annealing Technique for Enhanced Mobility in Smart Cities. Sensors (Basel); 16(7): 1013. July 2016.

[16] Qin Liuab, Jianmin Xua. Traffic Signal Timing Optimization for Isolated Intersections Based on Differential Evolution Bacteria Foraging Algorithm; Procedia-Social and Behavioral Sciences. Volume 43,Pages 210-215, 2012.

[17]Henrique Dezani, Norian Marranghello, Furio Damiani. Genetic algorithm-based traffic lights timing optimization and routes definition using Petri net model of urban traffic flow. IFAC Proceedings Volumes, Volume 47, Issue 3, 2014.

[18]F. van Wageningen-Kessels, H. van Lint, K. Vuik, and S. Hoogendoorn, "Genealogy of traffic flow models," EURO Journal on Transportation and Logistics, vol. 4, no. 4, pp. 445-473, Dec 2015.

[19]Chelouah, Rachid and Siarry, Patrick. (2000). A Continuous Genetic Algorithm Designed for the Global Optimization of Multimodal Functions. Journal of Heuristics. 6. 191-213. https://doi.org/10.1023/A:1009626110229. 